\title{
A Multivariate Threshold Varying Conditional Correlations Model
}

\author{
W. Kwan * W.K. $\mathrm{Li}^{\dagger} \quad$ K. $\mathrm{Ng}^{\ddagger}$
}

Revised September 26, 2006

\begin{abstract}
In this article, a multivariate threshold varying conditional correlation (TVCC) model is proposed. The model extends the idea of Engle (2002) and Tse \& Tsui (2002) to a threshold framework. This model retains the interpretation of the univariate threshold GARCH model and allows for dynamic conditional correlations. Techniques of model identification, estimation and model checking are developed. Some simulation results are reported on the finite sample distribution of the maximum likelihood estimate of the TVCC model. Real examples demonstrate the asymmetric behaviour of the mean and the variance in financial time series and the ability of the TVCC model to capture these phenomena.
\end{abstract}

Keywords: Multivariate TVCC model; Threshold; Conditional correlation; Volatility.

\section{Introduction}

During the last two decades, the modelling of conditional volatility in finance has been widely discussed in the literature. As a model for financial data with a changing conditional variance, Engle (1982) first proposed the autoregressive conditional heteroscedasticity (ARCH) model. Bollerslev (1986) extended this to a generalized ARCH (GARCH) model. Engle \& GonzálezRivera (1991) further extended the GARCH model to a semiparametric GARCH model which

\footnotetext{
*Email: ckwkwan@hku.hk Department of Statistics and Actuarial Science, The University of Hong Kong. $\dagger$ †mail: hrntlwk@hku.hk Department of Statistics and Actuarial Science, The University of Hong Kong

${ }_{\ddagger}^{\ddagger}$ Email: kaing@hku.hk Department of Statistics and Actuarial Science, The University of Hong Kong
} 
does not assume a parametric form of the noise distribution. A tremendous literature now exists for the GARCH model, for instance see Li, Ling \& McAleer (2002).

There have been lots of interest in nonlinear time series models, for instance, the selfexciting threshold autoregressive (SETAR) model of Tong $(1978,1980,1983)$ and Tong \& Lim (1980). Various tests for nonlinearity have since been developed. Keenan (1985) constructed a test for linearity which is an analogue of Tukey's one degree of freedom for nonadditivity test. Petruccelli (1986) proposed a portmanteau test for self-exciting threshold autoregressive nonlinearity model. Moreover, Tsay (1989) proposed an efficient procedure for testing threshold nonlinearity and successfully illustrated its use via the analysis of high-frequency financial data. During the time, many researchers have also extended the ARCH model to a nonlinear ARCH model, for example Li \& Lam (1995). Li \& Li (1996) extended the threshold ARCH model to a double-threshold ARCH model, which can handle the situation where both the conditional mean and the conditional variance specifications are piecewise linear given previous information. Pesaran \& Potter (1997) considered a floor and ceiling model for US output, which has a strong double threshold model flavour. Brooks (2001) further extended the double-threshold ARCH model to a double-threshold GARCH model.

After the development in univariate ARCH model, the study of multivariate ARCH models becomes the next important issue. Bollerslev, Engle and Wooldridge (1988) suggested a basic structure for a multivariate GARCH (MGARCH) model. Engle \& Kroner (1995) proposed a BEKK model which is a class of MGARCH model. Numerous applications of the multivariate GARCH models have been applied to financial data. For instance, Bollerslev (1990) studied the time-varying variance structure of the exchange rate in the European Monetary System. 
Kroner \& Claessens (1991) applied the models to evaluate the optimal debt portfolio in multiple currencies. Thereafter, Tsay (1998) proposed a procedure for testing multivariate threshold nonlinearity models and successfully illustrated its use via the analysis of monthly U.S. interest rates and two daily river flow series of Iceland. In order to satisfy the necessary conditions presented by Engle, Granger and Kraft (1984) for the conditional-variance matrix of an estimated MGARCH model to be positive definite, Bollerslev (1990) suggested a parsimonious constant-correlation MGARCH model. The necessary conditions for positive definiteness can be easily imposed during the optimization of the log-likelihood function. Engle \& Susmel (1993) investigated some international stock markets that have similar time-varying volatility. The recent work of Tse \& Tsui (2002) and Engle (2002) described a parsimonious MGARCH model that allows a time-varying correlation instead of a constant-correlation formulation for the conditional variance equation. A different time-varying conditional correlation GARCH model has been considered by Chan, Hoti \& McAleer (2004). Pelletier (2003) introduced a regime switching model of constant correlations within each regime. McAleer (2005) argued that the Tse \& Tsui (2002) approach is preferred because no re-standardization is necessary in the definition of the conditional correlation and adopted a Specific-to-General methodological approach to illustrate a number of important developments in the modelling of univariate and multivariate financial volatility. It is found that the time-varying correlation model could provide interesting and more realistic empirical results. As demonstrated by our empirical examples, there could be substantial difference in the correlational dynamics under different market situations. This difference could be significant enough in affecting a practitioner's decision on his forex portfolio.

In this paper, a multivariate threshold varying conditional correlation (TVCC) is proposed. 
The proposed model is an extension of the threshold approach for nonlinearity to the timevarying correlation model of Tse \& Tsui (2002). The conditional mean of the TVCC model follows a threshold vector autoregressive model. In Section 2, the construction of a multivariate TVCC model is discussed. A nonlinearity test for model building is presented in Section 3. Model identification and estimation procedures of the proposed model are given in Section 4 and Section 5. Here, model identification includes estimating the AR orders, GARCH orders, delay parameter and threshold parameter. Simulation results are provided in Section 6 . In Section 7, one empirical example of the proposed model using real data set is presented. It is the exchange rate data considered in Tse \& Tsui (2002). Finally some concluding remarks are given in the last section.

\section{A multivariate TVCC model}

In this section, multivariate threshold varying conditional correlation models are presented. Consider an $n$-dimensional multivariate time series $Z_{t}=\left(Z_{1 t}, \ldots, Z_{n t}\right)^{\prime}$, where $t=1, \ldots, T$. The conditional variance matrix of $Z_{t}$ follows a time-varying structure, $\operatorname{Var}\left(Z_{t} \mid F_{t-1}\right)=H_{t}=[h]_{i j, t}$, where $F_{t-1}$ is the information set $\left\{Z_{t-1}, \ldots, Z_{1}\right\}$ at time $t-1$. Rewrite $H_{t}=H_{t}^{\frac{1}{2}} H_{t}^{\frac{1}{2}}$, where

$H_{t}^{\frac{1}{2}}$ is the symmetric square-root matrix based on the spectral decomposition. Let $e_{t}=H_{t}^{\frac{1}{2}} \epsilon_{t}$, where $\epsilon_{t} \sim N(0, I)$. Here, $\epsilon_{t}=\left(\epsilon_{1 t}, \ldots, \epsilon_{n t}\right)^{\prime}$ is assumed to be independently distributed and $e_{t}=\left(e_{1 t}, \ldots, e_{n t}\right)^{\prime}$ is conditionally normally distributed with mean zero and variance-covariance matrix $H_{t}$. Here, $v^{\prime}$ denotes the transpose of $v$.

A multivariate threshold varying conditional correlations (TVCC) model is the main focus of this article. The present paper is an extension of the VC-MGARCH model of Tse \& Tsui 
(2002) using the threshold approach. This model will have an appealing property of dynamic correlations within a regime. In particular, the time varying conditional variance matrix $H_{t}$ is defined as follows: $H_{t}=D_{t} \Gamma_{t} D_{t}$. Define $D_{t}$ as a $n \times n$ diagonal matrix where the $i$ th diagonal element is $\sqrt{h_{i i, t}}$. Then, $\Gamma_{t}$ is the correlation matrix of $Z_{t}$. Let $l_{0}<l_{1}<\ldots<$ $l_{s-1}<l_{s}$ be a partition of the real line, where $l_{0}=-\infty$ and $l_{s}=\infty$. Let $d$ be the delay parameter and $r_{t-d}$ be a real-valued threshold variable. The $j$-th regime of a multivariate $\operatorname{TVCC}\left(p_{1}, \ldots, p_{s} ; P_{1}, \ldots, P_{s} ; Q_{1}, \ldots, Q_{s} ; s\right)$ model is given by

$$
\begin{aligned}
Z_{i, t} & =\Phi_{i, 0}^{(j)}+\sum_{k=1}^{p_{j}} \Phi_{i, k}^{(j)} Z_{i, t-k}+e_{i, t}, \\
h_{i i, t} & =c_{i}^{(j)}+\sum_{k=1}^{P_{j}} \alpha_{i, k}^{(j)} h_{i i, t-k}+\sum_{k=1}^{Q_{j}} \beta_{i, k}^{(j)} e_{i, t-k}^{2}, \quad l_{j-1}<r_{t-d} \leq l_{j}, \quad j=1, \ldots, s,
\end{aligned}
$$

where $c^{(j)}, \alpha_{i, k}^{(j)}$ and $\beta_{i, k}^{(j)}$, the parameters in the $j$-th regime, are non-negative and subject to

$$
\sum_{k=1}^{P_{j}} \alpha_{i, k}^{(j)}+\sum_{k=1}^{Q_{j}} \beta_{i, k}^{(j)}<1 .
$$

The above constraint is sufficient and involves only the $j$-th regime. Denote $L$ be the backshift operator, the $j$-th regime of the vector TVCC model can be defined as follows:

$$
\begin{aligned}
\Phi^{(j)}(L) Z_{t} & =e_{t}, \quad \text { with } \quad e_{t}=D_{t} \epsilon_{t} \\
H_{t} & =C^{(j)}+\sum_{k=1}^{P_{j}} A_{k}^{(j)} H_{t-k}+\sum_{k=1}^{Q_{j}} B_{k}^{(j)} \vec{e}_{t-k} \quad l_{j-1}<r_{t-d} \leq l_{j}, \quad j=1, \ldots, s,
\end{aligned}
$$

where $\vec{e}_{t}=\left(e_{1 t}^{2}, \ldots, e_{n t}^{2}\right)^{\prime}, C^{(j)}$ is a $n \times 1$ vector, and $A_{k}^{(j)}$ and $B_{k}^{(j)}$ are $k \times k$ matrices. The corresponding time-varying conditional correlation matrix $\Gamma_{t}$ in the $j$-th regime follows

$$
\Gamma_{t}=\left(1-\theta_{1}^{(j)}-\theta_{2}^{(j)}\right) \Gamma+\theta_{1}^{(j)} \Gamma_{t-1}+\theta_{2}^{(j)} \Psi_{t-1}
$$

where $\Gamma=\left\{\rho_{i j}\right\}$ is a time-invariant $n \times n$ positive definite symmetric matrix with unit diagonal elements and $\Psi_{t-1}$ is a $n \times n$ matrix whose elements are functions of the lagged standardized 
residuals $\hat{u}_{i, t}=\frac{e_{i, t}}{\sqrt{h_{i i, t}}}$. The parameters $\theta_{1}^{(j)}$ and $\theta_{2}^{(j)}$ are non-negative subject to $\theta_{1}^{(j)}+\theta_{2}^{(j)} \leq 1$. Denote $\Psi_{t}=\left\{\Psi_{i j, t}\right\}$. In Tse \& Tsui (2002), the matrix $\Psi_{t-1}$ follows

$$
\Psi_{i j, t-1}=\frac{\sum_{h=1}^{M} \hat{u}_{i, t-h} \hat{u}_{j, t-h}}{\sqrt{\left(\sum_{h=1}^{M} \hat{u}_{i, t-h}^{2}\right)\left(\sum_{h=1}^{M} \hat{u}_{j, t-h}^{2}\right)}}, \quad \text { for } M \geq n \text {. }
$$

Tse \& Tsui (2002) stated that $M \geq n$ is a necessary condition for $\Psi_{t-1}$ to be positive definite. Thus, $\Gamma_{t}$ would also be a positive definite correlation matrix with unit diagonal elements. As a result, $H_{t}$ is a positive-definite matrix and hence, $H_{t}^{\frac{1}{2}}$ is also a positive definite matrix. Let $\nu_{j}=\left(\Phi_{0}^{(j)^{\prime}}, \Phi_{1}^{(j)^{\prime}}, \ldots, \Phi_{p j}^{(j)^{\prime}}\right)^{\prime}, \alpha_{j}=\left(\alpha_{1}^{(j)^{\prime}}, \alpha_{2}^{(j)^{\prime}}, \ldots, \alpha_{P_{j}}^{(j)^{\prime}}\right)^{\prime}, \beta_{j}=\left(\beta_{1}^{(j)^{\prime}}, \beta_{2}^{(j)^{\prime}}, \ldots, \beta_{Q_{j}}^{(j)^{\prime}}\right)^{\prime}$ and $\theta_{j}=$ $\left(\theta_{1}^{(j)}, \theta_{2}^{(j)}\right)^{\prime}$, where $\Phi_{k}^{(j)}, \alpha_{k}^{(j)}$ and $\beta_{k}^{(j)}$ are the vectors of $\left(\Phi_{i, k}^{(j)}\right),\left(\alpha_{i, k}^{(j)}\right)$ and $\left(\beta_{i, k}^{(j)}\right)$ respectively. Denote $\lambda=\left(\nu^{\prime}, \alpha^{\prime}, \beta^{\prime}, \theta^{\prime}\right)^{\prime}$ and the true parameter vector be $\lambda_{0}$. The parameter space $\Lambda$ is assumed to be a compact subspace of Euclidean space such that $\lambda_{0}$ is an interior point in $\Lambda$. For the sake of the development of TVCC model, the assumptions in Li \& Li (1996) on threshold model, Ling \& McAleer (2003) on VARMA-GARCH and Chan, Hoti \& McAleer (2004) on GARCC are employed. They are summarized in the following regular conditions:

1. All the roots of $\left|\Phi^{(j)}(L)\right|=0$ lie outside the unit circle. $E\left(Z_{t} \mid F_{t-1}\right)$ possesses an $F_{t^{-}}$ measurable second-order stationary solution.

2. The matrix $\Gamma$ is a finite and $\rho(\Gamma)$ having a positive lower bound over $\Lambda$. All the element of $A_{i}^{(j)}$ and $B_{k}^{(j)}$ are nonnegative; each element of $C^{(j)}$ has positive lower and upper bounds over $\Lambda$; and all the roots of $\left|I_{k}-\sum_{i=1}^{P_{j}} A_{i}^{(j)} L^{i}-\sum_{k=1}^{Q_{j}} B_{k}^{(j)} L^{k}\right|=0$ are outside the unit circle.

3. The parameters $\theta_{1}^{(j)}$ and $\theta_{2}^{(j)}$ are nonnegative. 
4. $I_{k}-\sum_{i=1}^{P_{j}} A_{i}^{(j)} L^{i}$ and $\sum_{k=1}^{Q_{j}} B_{k}^{(j)} L^{k}$ are left coprime and satisfy other identifiability conditions given in Jeantheau (1998) (see also Dunsmuir \& Hannan, 1976).

Under conditions 1 - 3, by theorem 1 of Ling \& McAleer (2003), $\left\{Z_{t}, e_{t}, H_{t}\right\}$ are strictly stationary and ergodic. It is also assumed that in each regime there is at least one parameter with a value different from the values of the corresponding parameter in the other regime so that together with condition 4, the identification of the threshold model is ensured ( $\mathrm{Li} \& \mathrm{Li}, 1996)$. For simplicity of notation, the $\operatorname{TVCC}\left(p_{1}, \ldots, p_{s} ; P_{1}, \ldots, P_{s} ; Q_{1}, \ldots, Q_{s} ; s\right)$ will be rewritten as $\operatorname{TVCC}(p ; P ; Q ; s)$ if $p_{j}=p, P_{j}=P$ and $Q_{j}=Q$ for any $j=1, \ldots, s$. We may define the threshold structure separately for the conditional mean and the conditional variance. However, the present approach seems to be simpler in terms of presentation. If the threshold variable $r_{t-d}$ is the same for both mean and variance, then it can be shown that the two approaches are the same. As in Tse \& Tsui (2002), the number of parameters is parsimonious and also the conditional correlations are not restricted to be constants. The above models have $s$ regimes and are piecewise linear in the threshold space $r_{t-d}$. The time series will be nonlinear in time when $s$ is greater than 1. The threshold variable $r_{t-d}$ is assumed to be known, however the delay parameter $d$, the number of regimes $s$, and the threshold values $l_{j}$ are unknown. The TVCC model extends both Tong's (1990) threshold model and Tse \& Tsui's (2002) time varying multivariate generalized autoregressive conditional heteroscedasticity model in a natural way. It is shown in Tong \& Lim (1980) that the threshold model can capture various nonlinear phenomena. 


\section{A Threshold Nonlinearity Test}

A threshold nonlinearity test for multivariate GARCH time series models is proposed. The proposed test follows the idea of Tsay (1998), $H_{0}: s=1$ vs $H_{1}: s>1$. Suppose observations $\left\{Z_{t}\right\}$ are given, where $t=1, \ldots, T$. Consider the autoregression, $Z_{t}^{\prime}=Y_{t}^{\prime} \Phi+e_{t}^{\prime}, \quad t=\tau+$ $1, \ldots, T$, where $\tau=\max (p, d), Y_{t}=\left(\mathbf{1}^{\prime}, Z_{t-1}^{\prime}, \ldots, Z_{t-p}^{\prime}\right)^{\prime}$ is a $(p+1) n$-dimensional regressor and $\mathbf{1}$ is a $n \times 1$ vector of ones and $\boldsymbol{\Phi}$ denotes a parameter matrix. Define $S$ to be the set of values taken by the threshold variable $r_{t-d}$, i.e. $S=\left\{r_{\tau+1-d}, \ldots, r_{T-d}\right\}$. Let $r_{(k)}$ be the $k$-th smallest element of $S$, and $\mu(k)$ be the corresponding time index of $r_{(k)}$. The arranged autoregression based on the increasing order of $r_{t-d}$ is $Z_{\mu(k)+d}^{\prime}=Y_{\mu(k)+d}^{\prime} \Phi_{k}+e_{\mu(k)+d}^{\prime}, \quad k=1, \ldots, T-\tau$. where $\boldsymbol{\Phi}_{k}$ is the parameter matrix using data associated with the $k$-th smallest values of $r_{t-d}$.

Let $\hat{\boldsymbol{\Phi}}_{k}$ be the least squares estimate of $\boldsymbol{\Phi}_{k}$ of the arranged autoregression. Let $\hat{e}_{\mu(k+1)+d}=$ $Z_{\mu(k+1)+d}-\hat{\boldsymbol{\Phi}}_{k}^{\prime} Y_{\mu(k+1)+d}$ and $\hat{\xi}_{j, \mu(k+1)+d}=\hat{e}_{j, \mu(k+1)+d} / \sqrt{\hat{\sigma}_{j}^{2}+Y_{\mu(k+1)+d}^{\prime} U_{j, k} Y_{\mu(k+1)+d}}$ be respectively the predictive residual and the standardized predictive residual of the arranged autoregression, where $\hat{\sigma}_{j}^{2}=\sum_{i=1}^{k} \hat{e}_{j, \mu(i)+d}^{2} /(k-n p-1)$ is the residual mean squared error of the $j$ th element of $Z_{t}$ and $U_{j, k}=\left(\sum_{i=1}^{k} Y_{\mu(i)+d} Y_{\mu(i)+d}^{\prime}\right)^{-1}\left(\sum_{i=1}^{k} \hat{e}_{j, \mu(i)+d}^{2} Y_{\mu(i)+d} Y_{\mu(i)+d}^{\prime}\right)\left(\sum_{i=1}^{k} Y_{\mu(i)+d} Y_{\mu(i)+d}^{\prime}\right)^{-1}$.

Consider the regression $\hat{\xi}_{\mu(i)+d}^{\prime}=Y_{\mu(i)+d}^{\prime} \Psi+\eta_{\mu(i)+d}^{\prime}, \quad i=1, \ldots, T-\tau$, where $\hat{\xi}_{\mu(i)+d}$ is the vector $\left(\hat{\xi}_{j, \mu(i)+d}\right)$. The procedure is then to test $H_{0}: \Psi=0$ vs $H_{a}: \Psi \neq 0$. We consider as in Tsay (1998) the test statistic $R(d)=(T-\tau-(n p+1)) \times\left(\ln \left(\operatorname{det} A_{0}\right)-\ln \left(\operatorname{det} A_{1}\right)\right)$, where $d$, the delay parameter, indicates that the test depends on the threshold variable $r_{t-d}$,

$$
A_{0}=\frac{1}{T-\tau} \sum_{l=1}^{T-\tau} \hat{\xi}_{\mu(l)+d} \hat{\xi}_{\mu(l)+d}^{\prime} \quad \text { and } \quad A_{1}=\frac{1}{T-\tau} \sum_{l=1}^{T-\tau} \hat{\eta}_{\mu(l)+d} \hat{\eta}_{\mu(l)+d}^{\prime}
$$

and $\hat{\eta}_{t}$ is the least squares residual. Under the null hypothesis that $Z_{t}$ is linear, the standardised 
residuals $\xi_{t}$ converges almost surely to a martingale difference sequence with a homogeneous covariance matrix. Hence as in Tsay (1998, remark 2), by the functional central limit theorem (Helland 1982, Thm. 3.3), $(T-\tau) A_{0}$ and $(T-\tau) A_{1}$ of the test statistic $R(d)$ follow asymptotically Wishart distributions. Using the same argument as that in the multivariate multiple regression analysis (Johnson and Wichern 1998, p.308), $R(d)$ is asymptotically a chi-squared random variable with $n(n p+1)$ degrees of freedom (Tsay 1998, Thm. 2). Sometimes, threshold structure might not be found in the mean equation, we can then replace the $Z_{t}$ 's by the square of the residuals from a vector AR fit and repeat the above process (Li \& Li 1996). The squared residuals from the best-fitting AR model would be adopted in identifying the threshold structure of the conditional variance equation.

\section{Model Identification}

The next tasks to be carried out are model identification and parameter estimation which will be illustrated in this section and the next section respectively. For a simple linear AR model, model identification can be easily handled by examining the process of autocorrelation function (ACF) and partial autocorrelation function (PACF). However, when identifying a TVCC model, it will not be the case as autocorrelations are uninformative about asymmetry in the model. Arranged autoregressions are used as in Tsay (1989) for identifying the threshold model. In the previous section, procedures for testing the presence of threshold nonlinearity are given. Tsay (1989) pointed out that scatterplots of the arranged autoregressive estimates versus the specified threshold variable could provide useful information in locating the thresholds. A detailed discussion of the procedure would be given in the next section. Given the threshold 
variable, the AR orders of each regime can be identified by using the Akaike's information criterion (AIC).

Consider an AR-GARCH $(p ; P, Q)$ process, for simplicity, assuming the GARCH order $P$ and $Q$ are the same. An AR-GARCH process is a process $Z_{t}$ given by $Z_{i, t}=\Phi_{i, 0}+\sum_{k=1}^{p} \Phi_{i, k} Z_{i, t-k}+e_{i, t}$, with conditional variance given by $\sigma_{i, t}^{2}=c_{i}+\sum_{k=1}^{P} \alpha_{i, k} \sigma_{i, t-k}^{2}+\sum_{k=1}^{Q} \beta_{i, k} e_{i, t-k}^{2}$. Let $v_{i, t}=e_{i, t}^{2}-\sigma_{i, t}^{2}$. Then we have $e_{i, t}^{2}=c_{i}+\sum_{k=1}^{\operatorname{Max}(P, Q)}\left(\alpha_{i, k}+\beta_{i, k}\right) e_{i, t-k}^{2}+v_{i, t}-\sum_{k=1}^{P} \beta_{i, k} v_{i, t-k}$. Gouriéroux (1997, p.37) shows that $E\left(v_{i, t}-\sum_{k=1}^{P} \beta_{k} v_{i, t-k} \mid F_{t-1}\right)=0$. Therefore, the MGARCH model can be rewritten in an ARMA representation. This is useful in identifying the GARCH orders $P$ and $Q$ initially.

The overall identification procedure is as follows.

1. Select the AR order $p$ and the GARCH order $P, Q$. Usually, small lags for $P$ and $Q$ are common in empirical applications.

2. Fit arranged autoregressions for a given $p$ and each possible delays $d$, and perform the threshold nonlinearity test. When nonlinearity is detected, choose the delay parameter $d$ which maximizes the test statistics $R(d)$.

3. For given $p$ and $d$, locate the value of the threshold parameter by using Tsay' arranged autoregression based on the scatterplots of the elements of $\Phi$ versus the threshold variable.

4. If the threshold structure is identified, calculate the residuals $\hat{e}_{t}$ of the threshold AR model. Then fit the entire TVCC model.

5. Use an information criterion such as the AIC or Bayesian information criterion (BIC) to refine the AR orders, the GARCH orders, the delay and threshold parameters by repeating steps (1) - (4), if necessary. 
In step 1, the maximum AR order $p$ and GARCH order $P, Q$ may be selected by examining the $\mathrm{ACF}, \mathrm{PACF}$ and the ACF of the squared series. In step 2, the set of threshold delays $d$ may be $\{1,2, \ldots \max (p, P, Q)\}$. Note that in step 3 , the scatterplots of deterministic function of AR coefficient can be examined to locate the threshold parameters. Step 5 involves the refinement of the model that may rely on the AIC. The AIC has been used in the literature in selecting TAR models (Tong 1990). When all the parameters are fixed, the AIC is asymptotically equivalent to selecting the model having the smallest residual variance using the conditional least squares method (Tsay 1998).

\section{Estimation Details and Model Checking}

The specification of the threshold variable is a major issue in modelling threshold model, as it plays a key role in the nonlinear structure of the model. Assuming the order $p$ of the mean equation is known, Tsay (1998) indicates that the nonlinearity test will have good power when the delay $d$ is correctly specified. Following Tsay (1998), the delay parameter is estimated by the value $\hat{d}$ that provides the greatest value of $R(d)$ in the testing for threshold nonlinearity.

After obtaining the delay parameter, estimating the threshold values will be the next important issue. For ease of presentation, and without loss of generality, the case of $s=2$ is considered. Chan (1993) has shown the strong consistency of the estimator of a threshold model. In particular, the threshold value is super-consistent in the sense that, $\hat{l}=l+O_{p}(1 / N)$. We now propose a method for estimating the threshold values. For simplicity, the same threshold structure of the mean and conditional variance equations are considered. Extension to the case of different threshold structure for the mean and variance equation is direct. 
Under the arranged autoregression framework, the values of the arranged AR estimates become biased once the arranged autoregression crosses a threshold value. A scatterplot of the arranged AR estimates versus the threshold variable should reveal such changes in the AR estimates due to the bias and hence reveal also the locations of the threshold values. At each candidate threshold value, the AR coefficients in the first and second regime, $\Phi_{1}^{(1)}$ and $\Phi_{1}^{(2)}$ can be calculated respectively. However, the lag-1 AR coefficients have $n$ different values in each regime. In order to obtain a relevant scatterplot, we therefore have to consider a real valued deterministic function which can differentiate between $\Phi_{1}^{(1)}$ and $\Phi_{1}^{(2)}$. Here, the deterministic function is defined as the mean of the absolute least squares estimates of the lag1 AR coefficients. A scatterplot can then be obtained by plotting the values of the suggested deterministic function against the values of the threshold variable. As suggested in Tsay's (1989) if there is a threshold structure, a turning point would be observed around the true threshold. The threshold value can thus be estimated by the position with the greatest change of slope in the scatterplot. Given the threshold value, the conditional mean series becomes linear within each regime. Moreover, the threshold structure also applies to the conditional variance. The remaining task is to estimate the parameters. Denote $I_{j t}=I\left(l_{j-1}<r_{t-d} \leq l_{j}\right)$, where $I(\cdot)$ is the indicator function. Assuming normality, $e_{t} \mid F_{t-1} \sim N\left(0, H_{t}\right)$, the loglikelihood function (LLF), $L=\sum_{t=1}^{T} L_{t}$ can be obtained. The conditional loglikelihood at time $t, L_{t}$ is given by

$$
\begin{aligned}
L_{t} & =-\frac{1}{2} \sum_{j=1}^{s}\left(n \log 2 \pi+\log \left|H_{t}\right|+e_{t}^{\prime} H_{t}^{-1} e_{t}\right) I_{j t} \\
& =-\frac{1}{2} \sum_{j=1}^{s}\left(n \log 2 \pi+\log \left|\Gamma_{t}\right|+\sum_{i=1}^{n} \log \sigma_{i, t}^{2}+e_{t}^{\prime} D_{t}^{-1} \Gamma_{t}^{-1} D_{t}^{-1} e_{t}\right) I_{j t}
\end{aligned}
$$

The MLE of $\lambda, \hat{\lambda}$ is obtained by maximizing $L$ with respect to $\lambda$. Suppose that the threshold and delay parameters are known and that $\lambda_{0}$ is an interior point of a compact subset of Euclidean 
parameter space. Then, under the regularity conditions in this paper, the consistency of the QMLE follows from Thm. 3 of Chan, Hoti \& McAleer (2004) (see also Jeantheau 1998). Denote $G_{0}=-E_{\lambda_{0}}\left[\partial^{2} L / \partial \lambda \partial \lambda^{\prime}\right]$. Given $d$ and the threshold value, the model is piecewise linear. It is further assumed that

A1 . $\partial^{2} L / \partial \lambda \partial \lambda^{\prime}$ exists and is continuous in an open, convex neighbourhood of $\lambda_{0}$.

A2 . $\left.T^{-1}\left(\partial^{2} L / \partial \lambda \partial \lambda^{\prime}\right)\right|_{\lambda_{T}}$ converges to a finite non-singular matrix $G_{0}=T^{-1} E_{\lambda_{0}}\left(\partial^{2} L / \partial \lambda \partial \lambda^{\prime}\right)$ in probability for any sequence $\lambda_{T}$ such that $\hat{\lambda} \stackrel{p}{\rightarrow} \lambda_{0}$.

A3 . $\left.T^{-1 / 2}(\partial L / \partial \lambda)\right|_{\lambda_{0}} \stackrel{d}{\rightarrow} N\left(0, \Omega_{\lambda}\right)$, where $\Omega_{\lambda}=\lim T^{-1} E_{\lambda_{0}}\left[\left(\partial L_{t} / \partial \lambda\right)\left(\partial L_{t} / \partial \lambda^{\prime}\right)\right]$.

Denote $\Sigma_{0}=E_{\lambda_{0}}[\partial L / \partial \lambda]$. Following Chan, Hoti \& McAleer (2004), the asymptotic distribution of $\sqrt{n}\left(\hat{\lambda}-\lambda_{0}\right) \stackrel{d}{\rightarrow} N\left(0, \Sigma_{0}^{-1} G_{0} \Sigma_{0}^{-1}\right)$. If the conditional distribution of $e_{t}$ is normal, then $\operatorname{var}(\hat{\lambda})=$ $G_{0}^{-1}$ asymptotically.

In checking the adequacy of the ARMA models with homogeneous conditional covariance over time, residual autocorrelations has been widely applied. Li (1992) proposed the asymptotic distribution of residual autocorrelations of a general threshold nonlinear time series model. Li \& Mak (1994) provided the asymptotic distribution of squared residual autocorrelations of a general conditional heteroscedastic nonlinear time series model. Tse (2002) proposed an asymptotic distribution of his residual-based diagnostics for conditional heteroscedasticity models. However, the asymptotic covariances of the standardized residual autocorrelations and the squared residual autocorrelations are all very complicated. In order to simplify the complexity, Ling \& $\mathrm{Li}$ (1997) proposed and derived the asymptotic distribution of the lag $l$ sum of squared residual autocorrelations $\hat{R}_{l}$ of the model with $l=1, \ldots, M$. Here, the lag $l$ sum of squared residual 
autocorrelations of $i$-th regime, $\hat{R}_{l}^{(i)}$ is defined as

$$
\hat{R}_{l}^{(i)}=\frac{\sum_{k=1}^{n_{i}}\left(\hat{e}_{\mu_{i}(k)}^{\prime} \hat{H}_{\mu_{i}(k)}^{-1} \hat{e}_{\mu_{i}(k)}-\tilde{e}\right)\left(\hat{e}_{\mu_{i}(k)-l}^{\prime} \hat{H}_{\mu_{i}(k)-l}^{-1} \hat{e}_{\mu_{i}(k)-l}-\tilde{e}\right) I\left(\mu_{i}(k)-l \in S_{i}\right)}{\sum_{k=1}^{n_{i}}\left(\hat{e}_{\mu_{i}(k)}^{\prime} \hat{H}_{\mu_{i}(k)}^{-1} \hat{e}_{\mu_{i}(k)}-\tilde{e}\right)^{2}}
$$

with $\tilde{e}=\frac{1}{n_{i}} \sum_{k=1}^{n_{i}} \hat{e}_{\mu_{i}(k)}^{\prime} \hat{H}_{\mu_{i}(k)}^{-1} \hat{e}_{\mu_{i}(k)}$ where $n_{i}$ is the number of observations in the $i$-th regime; $\mu_{i}(k)$ denotes the time index of the $k$ th smallest threshold variable in the $i$ th regime and $S_{i}$ is the set of values of the time index of the threshold variable in $i$-th regime. Intuitively, $\hat{R}_{l}^{(i)}$ is the lag $l$ sum of squared residual autocorrelations within $i$ th regime. The statistic $Q(M)=n_{i} \sum_{l=1}^{M}\left[\hat{R}_{l}^{(i)}\right]^{2}$ is assumed to be asymptotically a chi-square random variable with $M$ degrees of freedom which is the correct asymptotic distribution when $\hat{e}_{t}$ are replaced by their population counter-part. Hence, $Q(M)$ can be used as a portmanteau statistic for testing the overall significance of $\hat{R}_{l}^{(i)}$ of the $i$-th regime. The empirical size and power of the statistic are studied in a simulation with 500 replications in the next section.

\section{Simulations}

Simulated realizations of the $\operatorname{TVCC}(1 ; 1 ; 1 ; 2)$ model are used to investigate the finite sample performance of the identification and estimation procedure in this section. In the simulation, 500 independent replications with sample sizes 1,000 and 2,000 of three different bivariate models are generated. The parameters in the simulation model are shown in Table 1 . In order to eliminate the simulation bias, the first $m_{0}$ observations generated will be deleted for ensuring the stability of the generated series. Tsay (1998) suggested $m_{0}=\lfloor 5 \sqrt{T}\rfloor$, where $T$ is the sample size. Therefore, the whole generated series length is $T+\lfloor 5 \sqrt{T}\rfloor$, with the first $5 \sqrt{T}$ observations 
discarded. As a result, the effect of the starting value, which is zero for all parameters, should be negligible. These choices work well in the study. For simplicity, the threshold structure of the mean equation and the conditional equation are the same. The threshold variable, $r_{t-d}$ is considered to be the first entry of the series with delay parameter equals to one. Also, the threshold value is set equal to zero and the value of $M$ is 6 .

As stated in Section 5, a real value $d$ deterministic function should be defined for differentiating between $\Phi_{1}^{(1)}$ and $\Phi_{1}^{(2)}$. In the estimation process, the deterministic function is the mean of the elements of $\Phi_{1}$. The average estimated results of the simulated models with sample sizes 1,000 and 2,000 are summarized in the tables below. The results are close to the true value. Values inside parenthesis and braces are the standard deviations and the bias of the estimates. The simulation was performed assuming that $d$ and the threshold variable are known. From Table 2 and Table 3, the estimates are in general fairly close to the true value with a small sample standard deviation. The estimates are closer to the true value while the standard deviations becomes smaller as the sample size gets larger. A quadratic loss function, $Q^{*}(\theta)=\sum(\hat{\theta}-\theta)^{2}$, is considered to compute the discrepancy of the Monte Carlo estimates, $\hat{\theta}$, from the true coefficient values, $\theta$. As expected, the values of the quadratic function for a longer series length are noticeably smaller in each simulation model. These results suggest that the estimates have small bias and standard errors. The result agrees with Chan (1993)'s strong consistency result on the estimators. The overall empirical size and empirical power of the statistic $Q(M)$ are also considered. The constant conditional variance with threshold vector autoregressive model is adopted as the null model for computing the empirical power. The proportion of rejections of the $Q(M)$ statistic, $M=6$, based on the upper fifth percentile of the corresponding asymptotic 
$\chi_{6}^{2}$ distribution is summarized in Table 2. Both empirical sizes and power seem acceptable.

\section{$7 \quad$ Empirical Results}

Empirical examples of the time-varying correlation multivariate threshold GARCH model are presented for one interesting series considered by Tse \& Tsui (2002). This set of data is transformed to first order differences of log value in percentage. The data set consists of two exchange rate (versus U.S. dollar) series, namely the Deutsche Mark and the Japanese Yen. There are 2,131 daily observations covering the period from January 1990 through June 1998.

Tse \& Tsui (2002) suggested a parsimonious AR order of the conditional mean equation to fit the data set. As there is a huge economic recession in Japan during the observed period, it is believed that changes in the relationship between the economic variables and the exchange rates may follow a threshold model. Let $Z_{1, t}$ be the Japanese Yen and $Z_{2, t}$ be the Deutsche Mark. From the graph in Tse \& Tsui (2002), it is found that the Deutsche Mark has a smaller variation than the Japanese Yen. Using $Z_{1, t-d}$ as the threshold variable, the proposed nonlinearity test is then performed. The asymptotic distribution of the $R(d)$ statistics is $\chi^{2}$ with $6 \mathrm{df}$. The nonlinearity test results, given in Table 5, clearly reject the linear hypothesis. Therefore, as expected, the test strongly suggests threshold nonlinearity. The delay parameter $d=1$ is chosen from $\{1,2,3,4\}$. Let the lagged values $(d=1)$ of Yen, $Z_{1, t-1}$ be the threshold variable. Using the mean of the estimated lag-1 AR parameter as the deterministic function, a scatterplot of the deterministic function against the threshold variable is given in Figure 1. Using the proposed method suggested in Section 5, the threshold value is estimated to be -0.0789. Refinement of AR orders and GARCH orders are achieved using AIC. A TVCC $(1 ; 1 ; 1 ; 2)$ model is identified and 
the estimated results are given in Table 5. According to the model checking procedure discussed in Section 5, the lag- $l$ sum of squared (standardized) residual autocorrelations are also given in Table 5 assuming $M=6$. There are 890 observations belonging to the first regime. It can be observed that the exchange rate data set has a double threshold structure.

During the observed period, the Japanese economy has been experiencing a great recession. The Japanese Yen had a huge drop and the volatility of the exchange rate market is high. Conditional correlations in each regime show a great fluctuation. From Table 5, it is found that $\theta_{2}$ in regime 2 is essentially zero but it is very significant in regime 1 . This demonstrates the usefulness of our TVCC model as the correlational dynamic changes according to regimes. To see this more clearly, the absolute rate of change of the conditional correlation coefficient is calculated whenever regime switching occurs. The results are now summarised under Fig 2(b). It shows that the change in regime is quite frequent and the rate of change is not a constant and with a maximum value of about 0.71 . It shows that the effect of $\Psi_{t-1}$ on $\Gamma_{t}$ is regime dependent and that the correlation $\rho$ between the Japanese Yen and the Deutsche Mark of the two regimes could be quite different. This difference may be big enough to affect a practitioner's decision on his forex portfolio. Note that volatility of the Deutsche Mark in regime 1 is larger than that in regime 2. It is also larger than the volatility of Yen in regime 1 but this situation reverses in regime 2. In Tables 5, it is shown that the total sum of the LLFs in the threshold model is greater than the LLF in the non-threshold model. Note that the diagnostic statistic of the threshold model for regime 1 and regime 2 are 3.03 and 5.52 respectively. Both values are not significant under the reference $\chi_{6}^{2}$ distribution with a $5 \%$ significant level. It is believed that the threshold model better represents the data. 


\section{Conclusion}

The model structure of the TVCC is an extension and a synthesis of the work of Tong $(1978,1980$, 1983, 1990), Tsay(1989, 1998), Tse \& Tsui (2002) and Engle (2002). The conditional variance matrix is positive definite and the conditional correlations are allowed to be non-constants. The number of parameters of the model is also parsimonious. A modelling methodology is proposed for the TVCC model. Extensions of Tsay's identification procedures are made to identify the AR orders, GARCH orders, delay parameters and threshold parameters. Some simulation results are presented. For empirical applications, the TVCC model is applied to the data sets in Tse \& Tsui (2002). The obtained TVCC models seem to capture well the threshold structure in the series. This suggests that the proposed TVCC model should be a potentially useful tool in modelling financial time series.

\section{Acknowledgments}

W.K. Li thanks the Hong Kong Research Grants Councils \& the Croucher Foundation for partial support of this research. We would also like to thank Professor Y.K. Tse for providing the financial time series data set. An earlier version of the paper was presented at the Symposium on Econometric Forecasting and High-Frequency Data Analysis May 7-8, 2004. The Symposium was jointly hosted by the Institute for Mathematical Sciences, National University of Singapore and the School of Economics and Social Sciences, Singapore Management University. The authors thank Professors R.F. Engle, Mike McAleer, Y.K. Tse, A. Tsui, two referees and the Editor for helpful discussions that led to improvement of the paper. 


\section{References}

[1] Bauwens, L., Lauren, S. and Rombouts, J.K.V. (2006), Multivariate GARCH models: a survey, Journal of Applied Econometrics, 21, pp. 79 - 109.

[2] Bollerslev, T. (1986), Generalized Autoregressive Conditional Heteroskedasticity, Journal of Econometrics, 31, pp. 307 - 327.

[3] Bollerslev, T. (1990), Modelling the Coherence in Short-Run Nominal Exchange Rates: A Multivariate Generalized ARCH Model, Review of Economics and Statistics, 72, pp. 498 505.

[4] Bollerslev, T., Engle, R.F. and Wooldridge, J.M. (1988), A Capital Asset Pricing Model with Time-varying Covariances, Journal of Political Economy, 96, No. 1, pp. 116 - 131.

[5] Brooks, C. (2001), A double-threshold GARCH model for the French Franc/Deutschmark exchange rate, Journal of Forecasting, 20, pp. 135 - 143.

[6] Chan, F., Hoti, S. and McAleer, M. (2004), Generalized Autoregressive Conditional Correlation, unpublished paper, School of Economics and Commerce, University of Western Australia.

[7] Chan, K.S. (1993), Consistency and Limiting Distribution of the Least Squares Estimator of a Threshold Autoregressive Model, Annals of Statistics, 21, 1, pp. 520 - 533.

[8] Dunsmuir, W. and E.J. Hannan (1976), Vector linear time series models. Advanced in Applied Probability, 8, pp. 339 - 364.

[9] Engle, R.F. (1982), Autoregressive Conditional Heteroscedasticity with Estimates of the Variance of United Kingdom Inflation, Econometrica, 50, pp. 987 - 1007.

[10] Engle, R.F. (2002), Dynamic Conditional Correlation: A simple Class of Multivariate Generalized Autoregressive Conditional Heteroskedasticity Models, Journal of Business and Economic Statistics, 20, 3, pp. 339 - 350.

[11] Engle, R.F. and González-Riveria, G. (1991), Semiparametric ARCH models, Journal of Business and Economic Statistics, 9, 4, pp. 345 - 359.

[12] Engle, R.F. and Kroner, K.F. (1995), Multivariate Simultaneous Generalized ARCH, Econometric Theory, 11, pp. 122 - 150.

[13] Engle, R.F. and Mezrich, J. (1996), GARCH for Groups, Risk, 9, pp. 36 - 40. 
[14] Engle, R.F. and Susmel, R. (1993), Common Volatility in International Equity Market, Journal of Business and Economic Statistics, 11, 2, pp. 167 - 176.

[15] Engle, R.F., Granger, C.W.J. and Kraft, D. (1984), Combining Competing Forecasts of Inflation Using a Bivariate ARCH model, Journal of Economic Dynamics and Control, 8, pp. 151 - 165 .

[16] Gouriéroux, C. (1997), ARCH Models and Financial Applications, Springer.

[17] Helland, I.S. (1982), Central Limit Theorems for Martingales with discrete or continuous time, Scandinavian Journal of Statistics, 9, pp. 79 - 94.

[18] Jeantheau, T. (1998), Strong consistency of estimators for multivariate ARCH models, Econometric Theory, 14, pp. 70 - 86.

[19] Johnson, R.A., and Wichern, D.W. (1998), Applied Multivariate statistical analysis (2nd ed.), Englewood Cliffs, NJ: Prentice Hall.

[20] Keenan, D.M. (1985), A Tukey nonadditivity-type test for time series nonlinearity, Biometrika, 72, 1, pp. 39 - 44.

[21] Kroner, K.F. and Claessens, S. (1991), Optimal Dynamic Hedging Portfolios and the Currency Composition of External Debt, Journal of International Money and Finance, 10, pp. 131 - 148.

[22] Lai, T.L. and Wei, C.Z. (1982), Least Squares Estimates in Stochastic Regression Models with Applications to Identification and Control of Dynamic Systems, The Annals of Statistics, 10, pp. $154-166$.

[23] Li, C.W. and Li, W.K. (1996), On a Double-Threshold Autoregressive Heteroscedastic Time Series Model, Journal of Applied Econometrics, Vol 11, pp. 253 - 274.

[24] Li, W.K. (1992), On the asymptotic standard errors of residual autocorrelations in nonlinear Time Series Modelling, Biometrika, 79, 2, pp. 435 - 437.

[25] Li, W.K. and Lam, K. (1995), Modelling asymmetry in stock returns by a threshold ARCH model, The Statistician, 44, 3, pp. 333 - 341.

[26] Li, W.K. and Mak, T.K. (1994), On the squared residual autocorrelations in conditional heteroskedastic variance by iteratively weighted least squares, Journal of Time Series Analysis, 15 , pp. $627-636$.

[27] Li, W.K., Ling, S. and McAleer M. (2002), Recent theoretical results for time series models with GARCH errors, Journal of Economic Surveys, 16, pp. 245 - 269. 
[28] Ling, S.Q. and Li, W.K. (1997), Diagnostic checking of Nonlinear Multivariate Time Series with Multivariate ARCH errors, Journal of Time Series Analysis, 18, pp. 447 - 464.

[29] Ling, S.Q. and McAleer, M. (2003), Asymptotic theory for a vector ARMA-GARCH model, Econometric Theory, 19, pp. 280 - 310.

[30] MacRae, E.C. (1974), Matrix derivatives with an application to an adaptive linear decision problem, The Annals of Statistics, 2, pp. 337 - 346.

[31] McAleer, M. (2005), Automated inference and learning in modeling financial volatility, Econometric Theory, 21, pp. $232-261$.

[32] Pelletier, D. (2003), Regime switching for dynamic correlations, unpublished manuscript, http://www4.ncsu.edu/ dpellet.

[33] Pesaran, M. H. and Potter, S. M. (1997), A floor and ceiling model of US output, Journal of Economic Dynamics and Control, 21, pp. 661 - 695.

[34] Petruccelli, J.D. and Davies, N. (1986), A portmanteau test for self-exciting threshold autoregressive-type nonlinearity in time series, Biometrika, 73, 3, pp. 687 - 694.

[35] Tong, H. (1978), On a threshold model, (ed. C.H. Chen), Pattern Recognition and Signal Processing, Sijthoff and Noordhoff, Amsterdam.

[36] Tong, H. (1980), A view on non-linear times series model building, Time series, (ed. O.D. Anderson), North Holland, Amsterdam.

[37] Tong, H. (1983), Threshold Models in Non-linear Time Series Analysis, Lecture Notes in Statistics, No. 21. Springer, Heidelberg.

[38] Tong, H. (1990), Non-Linear Time Series: A Dynamical System Approach, Oxford University Press, Oxford.

[39] Tong, H. and K.S. Lim (1980), Threshold autoregressive, limit cycles and cyclical data, Journal of the Royal Statistical Society, Series B, 42, pp. 245 - 292.

[40] Tsay, R.S. (1989), Testing and Modeling Threshold Autoregressive Processes, Journal of the American Statistical Association, March 1989, 84, No. 405, pp. 231 - 240.

[41] Tsay, R.S. (1998), Testing and Modeling Multivariate Threshold Models, Journal of the American Statistical Association, March 1998, 93, No. 443, pp. 1188 - 1202.

[42] Tse, Y.K. (2002), Residual-based diagnostics for conditional heteroscedasticity models, Econometrics Journal, 5, pp. 358 - 373. 


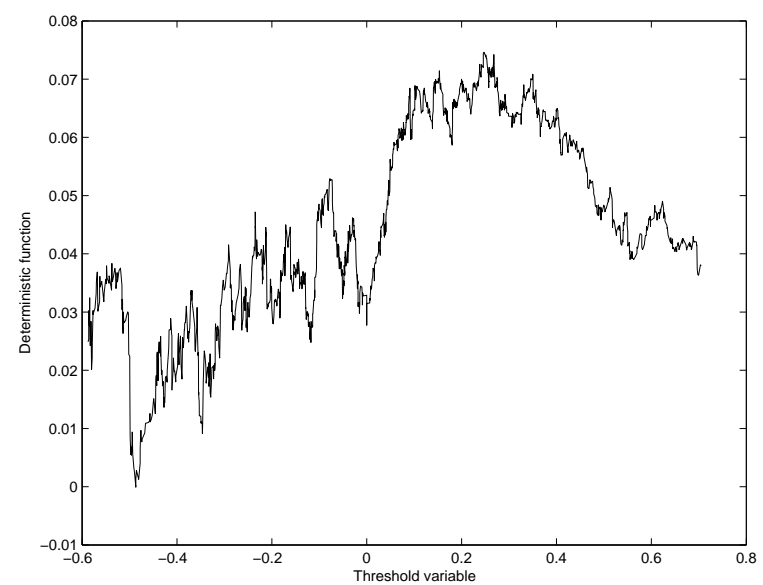

Figure 1: Scatterplot of deterministic function versus the threshold variable

Table 1: Parameters of the simulated models

\begin{tabular}{|c|c|c|c|c|c|c|c|c|}
\hline & \multicolumn{4}{|c|}{ model 1} & \multicolumn{4}{|c|}{ model 2} \\
\hline regime & \multicolumn{2}{|c|}{ first } & \multicolumn{2}{|c|}{ second } & \multicolumn{2}{|c|}{ first } & \multicolumn{2}{|c|}{ second } \\
\hline variable & 1 & 2 & 1 & 2 & 1 & 2 & & 2 \\
\hline$\phi_{0}$ & & 0.1 & 0.1 & 0.1 & 0.1 & 0.1 & 0.1 & 0.1 \\
\hline$\phi_{1}$ & 0.7 & 0.4 & 0.4 & 0.2 & 0.7 & 0.4 & 0.4 & 0.2 \\
\hline$C$ & 0.1 & 0.1 & 0.1 & 0.1 & 0.1 & 0.1 & 0.1 & 0.1 \\
\hline$\alpha$ & 0.8 & 0.8 & 0.6 & 0.6 & 0.8 & 0.8 & 0.6 & 0.6 \\
\hline$\beta$ & 0.1 & 0.1 & 0.1 & 0.1 & 0.1 & 0.1 & 0.1 & 0.1 \\
\hline$\theta_{1}$ & & & & & & & & \\
\hline$\theta_{2}$ & & & & & & & & \\
\hline$\rho$ & & & & & & & & \\
\hline Threshold & \multicolumn{4}{|c|}{0} & \multicolumn{4}{|c|}{0.2} \\
\hline
\end{tabular}

[43] Tse, Y.K. and Tsui, A.K.C. (2002), A multivariate GARCH Model with time-varying correlations, Journal of Business and Economic Statistics, July 2002, 20, No. 3, pp. 351 362.

[44] Wong, C.S. and Li, W.K. (2001), On a Mixture Autoregressive Conditional Heteroscedastic Model, Journal of the American Statistical Association, September 2001, 96, No. 455, pp. $982-995$.

[45] Wooldridge, J. M. (1991), On the application of robust, regression based diagnostics to models of conditional means and conditional covariances, Journal of Econometrics, 47(1), pp. 5 - 46 . 
Table 2: Diagnostic statistics with 500 replications

\begin{tabular}{|c|c|c|c|c|}
\hline \multirow[b]{3}{*}{ Regime } & \multicolumn{4}{|c|}{ Series length } \\
\hline & \multicolumn{2}{|c|}{1000} & \multicolumn{2}{|c|}{2000} \\
\hline & size & power & size & power \\
\hline First & 0.042 & 0.5982 & 0.038 & 0.6196 \\
\hline Second & 0.046 & 0.5776 & 0.044 & 0.5994 \\
\hline
\end{tabular}

Table 3: Estimation result from 500 simulated series with series length 1000

\begin{tabular}{|c|c|c|c|c|c|c|c|c|}
\hline & \multicolumn{4}{|c|}{ model 1} & \multicolumn{4}{|c|}{ model 2} \\
\hline \multirow{2}{*}{$\begin{array}{c}\text { regime } \\
\text { variable }\end{array}$} & \multicolumn{2}{|c|}{ first } & \multicolumn{2}{|c|}{ second } & \multicolumn{2}{|c|}{ first } & \multicolumn{2}{|c|}{ second } \\
\hline & 1 & 2 & 1 & 2 & 1 & 2 & 1 & 2 \\
\hline \multirow[t]{3}{*}{$\phi_{0}$} & 0.0968 & 0.0994 & 0.0888 & 0.0948 & 0.0973 & 0.1012 & 0.0911 & 0.0926 \\
\hline & $(0.0634)$ & $(0.0363)$ & $(0.0485)$ & $(0.0205)$ & $(0.0637)$ & $(0.0378)$ & $(0.0514)$ & $(0.0298)$ \\
\hline & $\{0.0032\}$ & $\{0.0006\}$ & $\{0.0112\}$ & $\{0.0052\}$ & $\{0.0027\}$ & $\{0.0012\}$ & $\{0.0089\}$ & $\{0.0074\}$ \\
\hline \multirow[t]{3}{*}{$\phi_{1}$} & 0.6855 & 0.3846 & 0.4106 & 0.2144 & 0.6868 & 0.3901 & 0.4081 & 0.2244 \\
\hline & $(0.0725)$ & $(0.0613)$ & $(0.0655)$ & $(0.0491)$ & $(0.0713)$ & $(0.0603)$ & $(0.0679)$ & $(0.0502)$ \\
\hline & $\{0.0145\}$ & $\{0.0154\}$ & $\{0.0106\}$ & $\{0.0144\}$ & $\{0.0132\}$ & $\{0.0099\}$ & $\{0.0081\}$ & $\{0.0244\}$ \\
\hline \multirow[t]{3}{*}{ C } & 0.1000 & 0.1028 & 0.0854 & 0.0824 & 0.1054 & 0.1070 & 0.0875 & 0.0827 \\
\hline & $(0.0478)$ & $(0.0466)$ & $(0.0314)$ & $(0.0176)$ & $(0.0485)$ & $(0.0486)$ & $(0.0327)$ & $(0.0313)$ \\
\hline & $\{0.0000\}$ & $\{0.0028\}$ & $\{0.0146\}$ & $\{0.0052\}$ & $\{0.0054\}$ & $\{0.0070\}$ & $\{0.0125\}$ & $\{0.0173\}$ \\
\hline \multirow[t]{3}{*}{$\alpha$} & 0.7652 & 0.7607 & 0.6167 & 0.6200 & 0.7638 & 0.7601 & 0.6236 & 0.6299 \\
\hline & $(0.0952)$ & $(0.0967)$ & $(0.0867)$ & $(0.0886)$ & $(0.0947)$ & $(0.0964)$ & $(0.0864)$ & $(0.0878)$ \\
\hline & $\{0.0348\}$ & $\{0.0393\}$ & $\{0.0167\}$ & $\{0.0200\}$ & $\{0.0362\}$ & $\{0.0399\}$ & $\{0.0236\}$ & $\{0.0299\}$ \\
\hline \multirow[t]{3}{*}{$\beta$} & 0.1018 & 0.0988 & 0.0965 & 0.0974 & 0.0992 & 0.0987 & 0.0932 & 0.0974 \\
\hline & $(0.0173)$ & $(0.0174)$ & $(0.0168)$ & $(0.0170)$ & $(0.0171)$ & $(0.0172)$ & $(0.0164)$ & $(0.0171)$ \\
\hline & $\{0.0018\}$ & $\{0.0012\}$ & $\{0.0035\}$ & $\{0.0026\}$ & $\{0.0008\}$ & $\{0.0013\}$ & $\{0.0068\}$ & $\{0.0026\}$ \\
\hline \multirow[t]{3}{*}{$\theta_{1}$} & \multirow{3}{*}{\multicolumn{2}{|c|}{$\begin{array}{c}0.2978 \\
(0.1412) \\
\{0.1022\}\end{array}$}} & & \multicolumn{2}{|c|}{0.2955} & \multirow{2}{*}{\multicolumn{2}{|c|}{$\begin{array}{c}0.2607 \\
(0.1552)\end{array}$}} \\
\hline & & & (0.1 & 46) & $(0.1$ & 19) & & \\
\hline & & & \multicolumn{2}{|c|}{0.1484} & \multicolumn{2}{|c|}{0.1045} & \multicolumn{2}{|c|}{0.1393} \\
\hline \multirow[t]{3}{*}{$\theta_{2}$} & \multicolumn{2}{|c|}{0.0828} & 0.0 & & \multirow{2}{*}{\multicolumn{2}{|c|}{$\begin{array}{c}0.0893 \\
(0.0116)\end{array}$}} & \multirow{2}{*}{\multicolumn{2}{|c|}{$\begin{array}{c}0.0894 \\
(00129)\end{array}$}} \\
\hline & \multirow{2}{*}{\multicolumn{2}{|c|}{$\begin{array}{l}(0.0113) \\
\{0.0172\}\end{array}$}} & \multicolumn{2}{|c|}{$(0.0195)$} & & & & \\
\hline & & & \multicolumn{2}{|c|}{0.0178} & \multicolumn{2}{|c|}{0.0107} & $\{0.1$ & $06\}$ \\
\hline$\rho$ & & 0.4 & & 0. & 86 & & 38 \\
\hline & $(0.0$ & 20) & $(0.0$ & 23) & (0. & 03) & (0. & 08) \\
\hline & $\{0.0$ & $54\}$ & $\{0.0$ & & $\{0.0$ & $86\}$ & $\{0$. & $38\}$ \\
\hline$l$ & & & & & & & & \\
\hline & & & 95) & & & & & \\
\hline & & $\{0.0$ & $36\}$ & & & $\{0.0$ & $36\}$ & \\
\hline$Q^{*}(\theta)$ & & 0.0 & & & & 0.0 & & \\
\hline
\end{tabular}

Standard deviations and bias of the estimates are included in the parentheses and the braces. 
Table 4: Estimation result from 500 simulated series with series length 2000

\begin{tabular}{|c|c|c|c|c|c|c|c|c|}
\hline & \multicolumn{4}{|c|}{ model 1} & \multicolumn{4}{|c|}{ model 2} \\
\hline \multirow{2}{*}{$\begin{array}{c}\text { regime } \\
\text { variable }\end{array}$} & \multicolumn{2}{|c|}{ first } & \multicolumn{2}{|c|}{ second } & \multicolumn{2}{|c|}{ first } & \multicolumn{2}{|c|}{ second } \\
\hline & 1 & 2 & 1 & 2 & 1 & 2 & 1 & 2 \\
\hline \multirow[t]{3}{*}{$\phi_{0}$} & 0.1004 & 0.0994 & 0.0922 & 0.0954 & 0.1006 & 0.1020 & 0.0932 & 0.0936 \\
\hline & $(0.0427)$ & $(0.0248)$ & (0.0387) & $(0.0221)$ & $(0.0451)$ & $(0.0253)$ & $(0.0384)$ & $(0.0227)$ \\
\hline & $\{0.0004\}$ & $\{0.0006\}$ & $\{0.0078\}$ & $\{0.0046\}$ & $\{0.0006\}$ & $\{0.0020\}$ & $\{0.0068\}$ & $\{0.0064\}$ \\
\hline \multirow[t]{3}{*}{$\phi_{1}$} & 0.6937 & 0.3850 & 0.4080 & 0.2113 & 0.6943 & 0.3938 & 0.4067 & 0.2201 \\
\hline & $(0.0502)$ & $(0.0436)$ & $(0.0500)$ & $(0.0375)$ & $(0.0509)$ & $(0.0418)$ & $(0.0490)$ & $(0.0417)$ \\
\hline & $\{0.0063\}$ & $\{0.0150\}$ & $\{0.0080\}$ & $\{0.0113\}$ & $\{0.0057\}$ & $\{0.0062\}$ & $\{0.0067\}$ & $\{0.0201\}$ \\
\hline \multirow[t]{3}{*}{$C$} & 0.0927 & 0.0962 & 0.0861 & 0.0853 & 0.0989 & 0.1032 & 0.0885 & 0.0851 \\
\hline & $(0.0375)$ & $(0.0379)$ & $(0.0234)$ & $(0.0249)$ & $(0.0377)$ & $(0.0392)$ & $(0.0243)$ & $(0.0253)$ \\
\hline & $\{0.0073\}$ & $\{0.0038\}$ & $\{0.0139\}$ & $\{0.0147\}$ & $\{0.0011\}$ & $\{0.0021\}$ & $\{0.0115\}$ & $\{0.0149\}$ \\
\hline \multirow[t]{3}{*}{$\alpha$} & 0.7775 & 0.7728 & 0.6118 & 0.6100 & 0.7743 & 0.7691 & 0.6136 & 0.6221 \\
\hline & $(0.0738)$ & $(0.0777)$ & $(0.0693)$ & $(0.0733)$ & $(0.0738)$ & $(0.0774)$ & $(0.0705)$ & $(0.0726)$ \\
\hline & $\{0.0225\}$ & $\{0.0272\}$ & $\{0.0118\}$ & $\{0.0100\}$ & $\{0.0257\}$ & $\{0.0309\}$ & $\{0.0136\}$ & $\{0.0221\}$ \\
\hline \multirow[t]{3}{*}{$\beta$} & 0.1017 & 0.0999 & 0.0969 & 0.0985 & 0.1006 & 0.1000 & 0.0936 & 0.0984 \\
\hline & $(0.0168)$ & $(0.0164)$ & $(0.0155)$ & $(0.0157)$ & $(0.0170)$ & $(0.0163)$ & $(0.0152)$ & $(0.0157)$ \\
\hline & $\{0.0017\}$ & $\{0.0001\}$ & $\{0.0031\}$ & $\{0.0015\}$ & $\{0.0006\}$ & $\{0.0000\}$ & $\{0.0064\}$ & $\{0.0016\}$ \\
\hline \multirow[t]{3}{*}{$\theta_{1}$} & & & \multicolumn{2}{|c|}{0.2617} & \multicolumn{2}{|c|}{0.3028} & \multicolumn{2}{|c|}{0.2652} \\
\hline & \multicolumn{2}{|c|}{$\begin{array}{c}0.3046 \\
(0.1413)\end{array}$} & \multicolumn{2}{|c|}{$(0.1477)$} & \multicolumn{2}{|c|}{$(0.1355)$} & \multicolumn{2}{|c|}{$(0.1492)$} \\
\hline & \multicolumn{2}{|c|}{0.0954} & \multicolumn{2}{|c|}{0.1383} & \multicolumn{2}{|c|}{0.0972} & \multicolumn{2}{|c|}{0.1348} \\
\hline \multirow[t]{3}{*}{$\theta_{2}$} & \multirow{2}{*}{\multicolumn{2}{|c|}{$\begin{array}{c}0.0897 \\
(0.0245)\end{array}$}} & \multicolumn{2}{|c|}{0.0894} & \multirow{2}{*}{\multicolumn{2}{|c|}{$\begin{array}{c}0.0910 \\
(0.0160)\end{array}$}} & 0. & \\
\hline & & & $(0$. & $38)$ & & & $(0.1$ & 38) \\
\hline & $\{0.0$ & $03\}$ & $\{0$. & $06\}$ & $\{0.0$ & $90\}$ & $\{0.1$ & $86\}$ \\
\hline$\rho$ & 0.5 & & & 69 & 0.5 & & & 01 \\
\hline & $(0.0$ & 43) & $(0.1$ & 91) & $(0.0$ & 30) & $(0.1$ & 75) \\
\hline & $\{0.0$ & $90\}$ & $\{0$. & $69\}$ & $\{0.0$ & $17\}$ & $\{0.1$ & $01\}$ \\
\hline$l$ & & & & & & & & \\
\hline & & & & & & & 5) & \\
\hline & & $\{0.0$ & $87\}$ & & & & $13\}$ & \\
\hline$Q^{*}(\theta)$ & & 0.0 & & & & 0.0 & & \\
\hline
\end{tabular}

Standard deviations and bias of the estimates are included in the parentheses and the braces. 
Table 5: Forex market data, Japanese Yen vs Deutsche Mark (TVCC(1;1;1;2))

\begin{tabular}{|c|c|c|c|c|c|c|c|c|c|}
\hline & \multicolumn{4}{|c|}{ Estimation } & \multicolumn{3}{|c|}{ Diagnostic } & \multirow{2}{*}{\multicolumn{2}{|c|}{$\begin{array}{c}\text { Nonlinearity } \\
\text { test }\end{array}$}} \\
\hline regime & \multicolumn{2}{|c|}{ first } & \multicolumn{2}{|c|}{ second } & & first & second & & \\
\hline variable & $\mathrm{J}$ & $\mathrm{D}$ & $\mathrm{J}$ & $\mathrm{D}$ & $k$ & {$\left[\hat{R}_{k}^{(1)}\right]^{2}$} & {$\left[\hat{R}_{k}^{(2)}\right]^{2}$} & $d$ & $R(d)$ \\
\hline$\phi_{0}$ & $\begin{array}{c}0.0400 \\
(0.0308)\end{array}$ & $\begin{array}{c}0.0149 \\
(0.0132)\end{array}$ & $\begin{array}{c}-.0013 \\
(0.0024)\end{array}$ & $\begin{array}{c}0.0067 \\
(0.0022)\end{array}$ & 1 & $4.24 \times 10^{-3}$ & $2.75 \times 10^{-5}$ & 1 & 292.71 \\
\hline$\phi_{1}$ & $\begin{array}{c}0.0518 \\
(0.0474)\end{array}$ & $\begin{array}{c}0.0570 \\
(0.0341)\end{array}$ & $\begin{array}{c}0.0388 \\
(0.0041)\end{array}$ & $\begin{array}{c}0.0235 \\
(0.0111)\end{array}$ & 2 & $3.86 \times 10^{-3}$ & $2.57 \times 10^{-3}$ & 2 & 288.75 \\
\hline$C$ & $\begin{array}{c}0.0325 \\
(0.0173)\end{array}$ & $\begin{array}{c}0.0140 \\
(0.0042)\end{array}$ & $\begin{array}{c}0.0001 \\
(0.0238)\end{array}$ & $\begin{array}{c}0.0102 \\
(0.0091)\end{array}$ & 3 & $4.31 \times 10^{-3}$ & $2.57 \times 10^{-4}$ & 3 & 229.35 \\
\hline$\alpha$ & $\begin{array}{c}0.9083 \\
(0.0316)\end{array}$ & $\begin{array}{c}0.9627 \\
(0.1069)\end{array}$ & $\begin{array}{c}0.9227 \\
(0.0637)\end{array}$ & $\begin{array}{c}0.8838 \\
(0.0386)\end{array}$ & 4 & $5.08 \times 10^{-4}$ & $1.83 \times 10^{-4}$ & 4 & 249.04 \\
\hline$\beta$ & $\begin{array}{c}0.0409 \\
(0.0124)\end{array}$ & $\begin{array}{c}0.0363 \\
(0.0109)\end{array}$ & $\begin{array}{c}0.0700 \\
(0.0107)\end{array}$ & $\begin{array}{c}0.0737 \\
(0.0351)\end{array}$ & 5 & $8.54 \times 10^{-5}$ & $2.74 \times 10^{-5}$ & & \\
\hline$\theta_{1}$ & & $\begin{array}{l}80 \\
06)\end{array}$ & $\begin{array}{r}0.9 \\
(0.0\end{array}$ & 66 & 6 & $5.94 \times 10^{-4}$ & $5.89 \times 10^{-4}$ & & \\
\hline$\theta_{2}$ & & $\begin{array}{l}58 \\
73)\end{array}$ & $\begin{array}{r}0.0 \\
(0.0\end{array}$ & $\begin{array}{l}01 \\
97)\end{array}$ & & & & & \\
\hline$\rho$ & & & $\begin{array}{r}0.5 \\
(0.1\end{array}$ & $\begin{array}{l}48 \\
68)\end{array}$ & & & & & \\
\hline$l$ & & -0.0 & 789 & & & & & & \\
\hline LLF & -1.7 & $\times 10^{3}$ & -1.93 & $\times 10^{3}$ & & & & & \\
\hline
\end{tabular}

Standard errors of estimated are included in the parentheses.

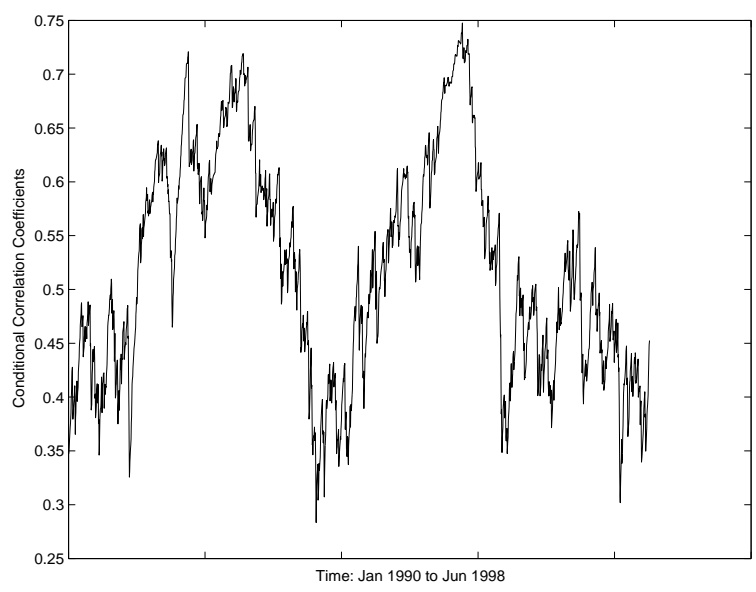

Fig.2(a)

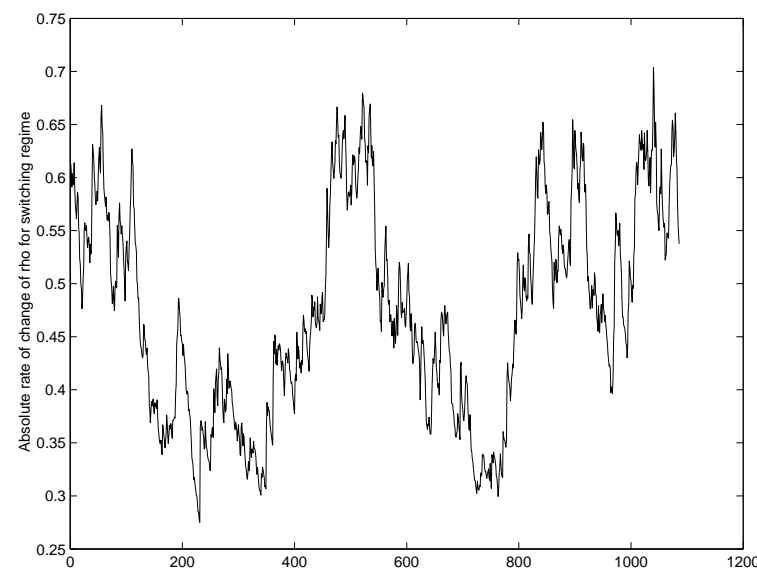

Fig.2(b) 